\title{
Spherical cavity in the layer subjected to dynamic load
}

\author{
Piotr Koziol ${ }^{1,1}$, Cristinel Mares ${ }^{2}$, and Rafat Pilecki ${ }^{3}$ \\ ${ }^{1}$ Cracow University of Technology, Faculty of Civil Engineering, Kraków, Poland \\ ${ }^{2}$ Brunel University, College of Engineering, Design and Physical Sciences, Mechanical and \\ Aerospace Engineering, London, UK \\ ${ }^{3}$ Former student of Cracow University of Technology
}

\begin{abstract}
The problem of wave motion with spherical symmetry is analysed. For this purpose, a spherical cavity surrounded by layer is considered as a mathematical model which can be used for modelling various phenomena in solid mechanics. The additional layer is also spherical and the outer space is described as unbounded medium. All layers are isotropic, homogeneous and linearly elastic, although the presented formulation allows inclusion of weak nonlinearities. Analytical solutions for displacement and stresses (radial and circumferential) are presented along with some discussion of possible model extensions.
\end{abstract}

Keywords: spherical cavity, dynamic load, wave propagation

\section{Introduction}

The problem presented in this paper is associated with geotechnical aspects, in particular with micro explosions in solid, e.g. micro blasting technology related to soil consolidation. Similar methods can be used to improve foundation of linear structures, such as highways or railways. For this reason, it is important for safety to predict how waves propagate outside working area to outer space surrounding constructions. Analysis of foundation behaviour at a very close distance from the source of explosion is of importance for a proper recognition of soil condition after the whole improvement process and for planning the effectiveness of the engineering method itself. This paper does not concentrate on technical aspects of geotechnical technology but it proposes simplified model analysis along with analytical solution for displacement (wave motion) and stresses produced by a load acting on walls of the spherical cavity $[1,2]$. This solution might be helpful in deeper analysis of dynamic behaviour of such structures. It also allows several extensions, including nonlinear properties of solid, leading to semi-analytical solutions obtained by using already verified semi-

\footnotetext{
${ }^{1}$ Corresponding author: pkoziol@pk.edu.pl
} 
analytical approximations based on Adomian's decomposition and wavelet estimations using coiflet expansions of functions [3-7].

\section{Model formulation}

The mathematical model considered in this paper consists of spherical cavity placed in outer unbounded solid. This layer can be also surrounded by one or several symmetric layers with varying geometrical and physical properties $[1,2]$.

The cross section of such structure with two additional layers is shown in figure 1.

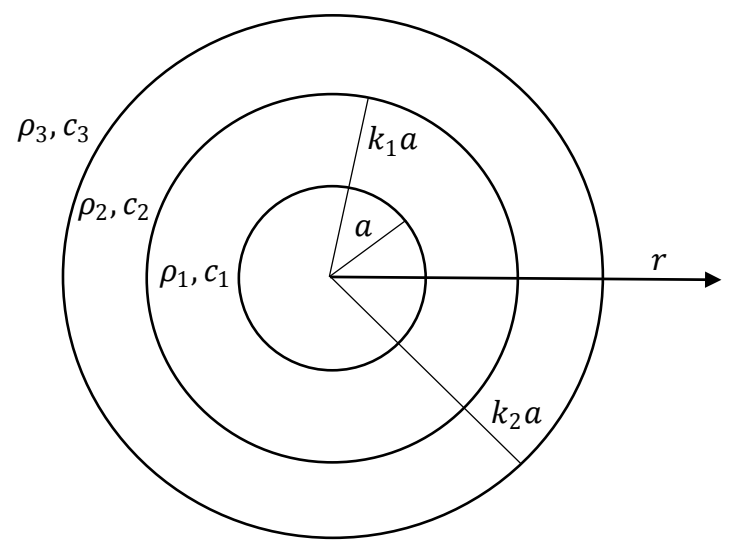

Fig. 1. Cross section of the spherical cavity with finite symmetrical layer around it, placed in the unbounded outer medium.

The notation used in this figure means the following:

$a$ - radius of symmetrical cavity;

$r$ - direction of wave propagation (distance from the centre of cavity);

$k_{1} a, k_{2} a$-outer radius of the first and the second layers surrounding spherical cavity $\left(k_{1}<\right.$ $k_{2}$ are real numbers greater than 1 );

$\rho_{1}, \rho_{2}, \rho_{3}-$ mass densities of layers;

$c_{1}, c_{2}, c_{3}$ - phase velocities of longitudinal wave in corresponding layers.

One can consider the following equation of motion in unbounded medium with spherical symmetry in $r$ direction [1] (see Fig. 2)

$$
\frac{\partial \tau_{r}}{\partial r}+\frac{2}{r}\left(\tau_{r}-\tau_{\theta}\right)=\rho \frac{\partial^{2} u}{\partial t^{2}}
$$

with nonzero stresses and displacement $u(r, t)[\mathrm{m}]$, where volumetric forces are neglected and the applied load possesses the required symmetry at all times.

The radial stress $\tau_{r}\left[\frac{\mathrm{N}}{\mathrm{m}^{2}}\right]$ (parallel to radius $r$ ) and the circumferential stress $\tau_{\theta}\left[\frac{\mathrm{N}}{\mathrm{m}^{2}}\right]$ (perpendicular to radius $r$ ) are defined as follows [1]:

$$
\tau_{r}=(\lambda+2 \mu) \frac{\partial u}{\partial r}+2 \lambda \frac{u}{r}
$$

and

$$
\tau_{\theta}=\lambda \frac{\partial u}{\partial r}+2(\lambda+\mu) \frac{u}{r}
$$


where $\rho\left[\frac{\mathrm{kg}}{\mathrm{m}^{3}}\right], t[\mathrm{~s}], \lambda\left[\frac{\mathrm{N}}{\mathrm{m}^{2}}\right]$ and $\mu\left[\frac{\mathrm{N}}{\mathrm{m}^{2}}\right]$ are the mass density, the time and Lame elastic constants, respectively. One should note that tangential stresses are zero due to the spherical symmetry.

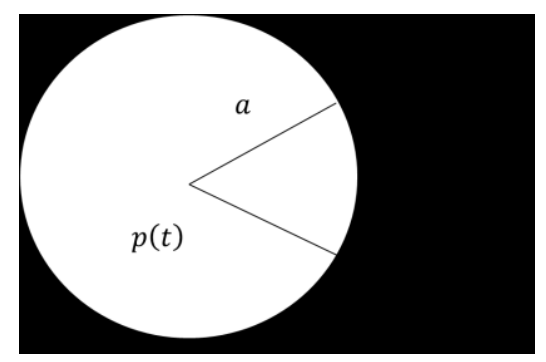

Fig. 2. Scheme of spherical cavity with radius $a$ and a load $p(t)\left[\frac{\mathrm{N}}{\mathrm{m}^{2}}\right]$ acting on internal walls of cavity. Using the following initial and boundary conditions:

$$
\tau_{r}=-p(t) \text { for } \quad r=a, t \geq 0
$$

and

$$
u(r, t)=\frac{\partial u(r, t)}{\partial t}=0 \quad \text { for } \quad r \geq a, t<0
$$

the system of equations (1-3) can be solved by introducing potential theory.

\section{Solution}

The solution for spherical cavity, described in this section, is presented in [1], whereas section 4 contains its extension to more complex model with additional layers surrounding cavity and forms a novelty of study shown in present paper. Substituting equations (2) and (3) to (1) leads to the following partial differential equation of motion:

$$
\frac{\partial^{2} u}{\partial r^{2}}+\frac{2}{r} \frac{\partial u}{\partial r}-\frac{2 u}{r^{2}}=\frac{1}{c^{2}} \frac{\partial^{2} u}{\partial t^{2}}
$$

For solving equation (6) one can use displacement potential function $\phi(r, t)$ fullfiling the condition

$$
u=\frac{\partial \phi}{\partial r}
$$

along with new variable

$$
s=t-\frac{r-a}{c}
$$

where $\mathrm{c}$ is a phase velocity of longitudinal wave in medium. Then new formulas depending on the variable $s$ can be derived for displacement and stresses:

$$
\begin{gathered}
u(r, t)=-w^{\prime}(s) \frac{1}{c r}-w(s) \frac{1}{r^{2}} \\
\tau_{r}(r, t)=\frac{\rho c^{2}}{1-v}\left[\frac{(1-v) w^{\prime \prime}}{c^{2} r}+2(1-2 v)\left(\frac{w^{\prime}}{c r^{2}}+\frac{w}{r^{3}}\right)\right] \\
\tau_{\theta}(r, t)=\frac{\rho c^{2}}{1-v}\left[\frac{v w^{\prime \prime}}{c^{2} r}-(1-2 v)\left(\frac{w^{\prime}}{c r^{2}}+\frac{w}{r^{3}}\right)\right]
\end{gathered}
$$

where the function 


$$
w(s)=r \phi(r, t)
$$

is a solution representing outgoing wave and $v$ denotes the Poisson's ratio [1].

One should note that the function (7) is solution of equation (6) if the function (12) is solution of the following equation of motion [1]

$$
\frac{\partial^{2} \phi(r, t)}{\partial r^{2}}=\frac{1}{c^{2}} \frac{\partial^{2} \phi(r, t)}{\partial t^{2}}
$$

By applying initial and boundary conditions (4-5) one obtains the ordinary differential equation for $w(s)$

$$
\frac{d^{2} w}{d s^{2}}+2 \alpha \frac{d w}{d s}+\left(\alpha^{2}+\beta^{2}\right) w=-p(s) \frac{a}{\rho}
$$

where the coefficients $\alpha$ and $\beta$ are defined as follows:

$$
\begin{gathered}
\alpha=\frac{1-2 v}{1-v} \frac{c}{a} \\
\beta^{2}=\frac{1-2 v}{(1-v)^{2}} \frac{c^{2}}{a^{2}} .
\end{gathered}
$$

By substituting

$$
w(s)=g(s) \exp (-\alpha s)
$$

to equation (14) one can reduce it to the following form:

$$
\frac{d^{2} g}{d s^{2}}+\beta^{2} g=-\frac{a}{\rho} p(s) \exp (\alpha s) .
$$

Hence, by applying the boundary conditions and the Green's function theory, one can obtain solution for unbounded layer:

$$
g(s)=-\frac{a}{\rho} \frac{1}{\beta} \int_{0}^{s} p(\tau) \exp (\alpha \tau) \sin [\beta(s-\tau)] d \tau
$$

which allows to effectively compute displacement and stresses for given physical properties of medium and particular load representations.

One should note that initial and boundary conditions (4-5) give

$$
w(0)=w^{\prime}(0) \equiv 0 \text {. }
$$

\section{Model extension and computational examples}

The obtained solution (19) allows parametrical analysis of the system with several possible cases. These include e.g. a number of layers surrounding spherical cavity or different forms of load $p(t)$. To show advantages of the developed procedure, the case of constant pressurization represented by the Heaviside function and one spherical layer with finite thickness, which surrounds the cavity placed in the unbounded medium is analysed (see Fig. 3).

Using the procedure presented in section 3 one can derive solution for the bounded layer (with index 1)

$$
g_{1}\left(s_{1}\right)=-\frac{a}{\rho_{1}} \int_{0}^{s_{1}} G\left(s_{1}-\tau\right) p(\tau) \exp \left(\alpha_{1} \tau\right) d \tau
$$

and the unbounded medium (with index 2)

$$
g_{2}\left(s_{2}\right)=A_{1} \sin \left(\beta_{2} s_{2}\right)+A_{2} \cos \left(\beta_{2} s_{2}\right)-\frac{a}{\rho_{2}} \int_{0}^{s_{2}} G\left(s_{2}-\tau\right) p(\tau) \exp \left(\alpha_{2} \tau\right) d \tau,
$$


respectively. $G(s)$ denotes the one-sided Green's function for the operator

$$
\frac{d^{2}}{d s_{2}^{2}}+\beta_{2}^{2}
$$

(comp. equation 18). $A_{1}$ and $A_{2}$ are constants which should be found on the basis of the boundary conditions.

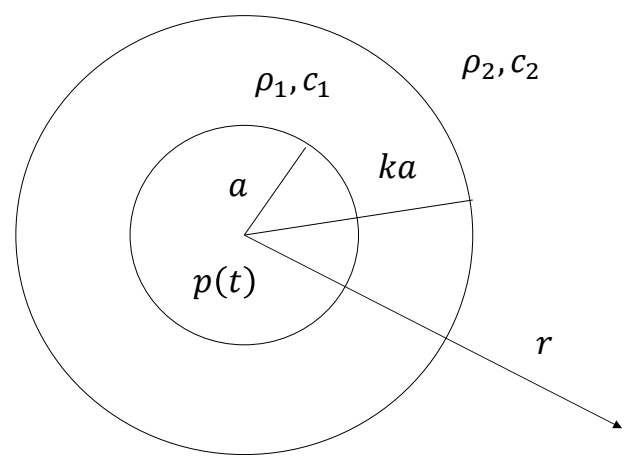

Fig. 3. Cross section scheme of spherical cavity surrounded by layer with finite thickness.

Solution of the problem can be found then by introducing continuity conditions for displacements and stresses

$$
u_{1}(k a, t)=u_{2}(k a, t), u_{1}{ }^{\prime}(k a, t)=u_{2}{ }^{\prime}(k a, t)
$$

i.e.

$$
\begin{aligned}
u_{1}(r, t)= & {\left[\left(\frac{a}{\rho_{1} \beta_{1} r^{2}}-\frac{\alpha_{1} a}{c_{1} \rho_{1} \beta_{1} r}\right) \int_{0}^{s_{1}} \sin \left(\beta_{1}\left(s_{1}-\tau\right)\right) p(\tau) \exp \left(\alpha_{1} \tau\right) d \tau+\right.} \\
& \left.\frac{a}{c_{1} \rho_{1} r} \int_{0}^{s_{1}} \cos \left(\beta_{1}\left(s_{1}-\tau\right)\right) p(\tau) \exp \left(\alpha_{1} \tau\right) d \tau\right] \cdot \exp \left(-\alpha_{1} s_{1}\right)
\end{aligned}
$$

for the finite layer and

$$
\begin{gathered}
u_{2}(r, t)=\left[\left(\left(\frac{\alpha_{2}}{c_{2} r}-\frac{1}{r^{2}}\right) \cos \left(\beta_{2} s_{2}\right)+\frac{\beta_{2}}{c_{2} r} \sin \left(\beta_{2} s_{2}\right)\right) \cdot A_{1}+\right. \\
\left(\left(\frac{\alpha_{2}}{c_{2} r}-\frac{1}{r^{2}}\right) \sin \left(\beta_{2} s_{2}\right)-\frac{\beta_{2}}{c_{2} r} \cos \left(\beta_{2} s_{2}\right)\right) \cdot A_{2}+ \\
\left.\left(\frac{a}{\rho_{2} \beta_{2} r}\left(\frac{\alpha_{2}}{c_{2} r}-\frac{1}{r^{2}}\right)+\frac{a}{c_{2} \rho_{2} r}\right) \int_{0}^{s_{2}} \sin \left(\beta_{2}\left(s_{2}-\tau\right)\right) p(\tau) \exp \left(\alpha_{2} \tau\right) d \tau\right] \cdot \exp \left(-\alpha_{2} s_{2}\right)
\end{gathered}
$$

for the unbounded medium, respectively.

Introducing dimensionless variables and parameters

$$
\bar{t}=\frac{t c_{1}}{a}, d=\frac{r}{a}, l=\frac{c_{2}}{c_{1}}, l_{1}=\frac{\rho_{2}}{\rho_{1}}, \bar{u}=\frac{u}{a}, \bar{\tau}_{r}=\frac{\tau_{r}}{p_{0}}, \bar{\tau}_{\theta}=\frac{\tau_{\theta}}{p_{0}}
$$

allows to carry out effective parametrical analysis of the considered system.

In the case of load represented by constant pressurization described by the Heaviside function

$$
p(t)=p_{0} H(t)
$$


the constants $A_{1}$ and $A_{2}$ take the form

$$
A_{1}=\frac{p_{0} a^{3}}{c_{1}{ }^{2} \rho_{1}} \cdot \tilde{A}_{1}\left(l, l_{1}, k\right)=M \cdot a^{3} \cdot \tilde{A}_{1}\left(l, l_{1}, k\right)
$$

and

$$
A_{2}=\frac{p_{0} a^{3}}{c_{1}^{2} \rho_{1}} \cdot \tilde{A}_{2}\left(l, l_{1}, k\right)=M \cdot a^{3} \cdot \tilde{A}_{2}\left(l, l_{1}, k\right) .
$$

Then the sought solution can be written as:

$$
\begin{gathered}
\bar{u}_{1}(d, \bar{t})=\frac{3}{8} M\left(\frac{1}{d}\right)^{2}\left[\exp \left(-\frac{2}{3}(\bar{t}-d+1)\right) \cdot\right. \\
\left(-2 \sqrt{2} d \sin \left(\frac{2 \sqrt{2}}{3}(\bar{t}-d+1)\right)+\cos \left(\frac{2 \sqrt{2}}{3}(\bar{t}-d+1)\right)+\right. \\
\left.\sin \left(\frac{2 \sqrt{2}}{3}(\bar{t}-d+1)\right)\right] \\
\bar{u}_{2}(d, \bar{t})=-\frac{1}{24} M\left(\frac{1}{d}\right)^{2}\left[\exp \left(-\frac{2}{3}(l \bar{t}-d+1)\right) \cdot\right. \\
\left(\tilde{A}_{1}\left(l, l_{1}, k\right) \cos \left(\frac{2 \sqrt{2}}{3}(l \bar{t}-d+1)\right)+\right. \\
\left.\left.\tilde{A}_{2}\left(l, l_{1}, k\right) \sin \left(\frac{2 \sqrt{2}}{3}(l \bar{t}-d+1)\right)\right)-18 \frac{1}{l^{2} l_{1}}\right]
\end{gathered}
$$

for displacements and

$$
\begin{gathered}
\bar{\tau}_{r, 1}(d, \bar{t})=-\frac{3}{8}\left(\frac{1}{d}\right)^{3}\left[\operatorname { e x p } ( - \frac { 2 } { 3 } ( l \overline { t } - d + 1 ) ) \cdot \left(B_{r, 1}{ }^{(1)} \cos \left(\frac{2 \sqrt{2}}{3}(l \bar{t}-d+1)\right)+\right.\right. \\
\left.\left.B_{r, 1}{ }^{(2)} \sin \left(\frac{2 \sqrt{2}}{3}(l \bar{t}-d+1)\right)-2\right)\right] \\
\bar{\tau}_{\theta, 1}(d, \bar{t})=-\frac{1}{8}\left(\frac{1}{d}\right)^{3}\left[\operatorname { e x p } ( - \frac { 2 } { 3 } ( l \overline { t } - d + 1 ) ) \cdot \left(B_{\theta, 1}{ }^{(1)} \cos \left(\frac{2 \sqrt{2}}{3}(l \bar{t}-d+1)\right)+\right.\right. \\
\left.\left.B_{\theta, 1}{ }^{(2)} \sin \left(\frac{2 \sqrt{2}}{3}(l \bar{t}-d+1)\right)-4\right)\right] \\
\bar{\tau}_{r, 2}(d, \bar{t})=-\frac{1}{12}\left(\frac{1}{d}\right)^{2}\left[\operatorname { e x p } ( - \frac { 2 } { 3 } ( l \overline { t } - d + 1 ) ) \cdot \left(B_{r, 2}{ }^{(1)} \cos \left(\frac{2 \sqrt{2}}{3}(l \bar{t}-d+1)\right)+\right.\right. \\
\left.\left.B_{r, 2}{ }^{(2)} \sin \left(\frac{2 \sqrt{2}}{3}(l \bar{t}-d+1)\right)\right)+\frac{12}{d l^{2} l_{1}}\right] \\
\bar{\tau}_{\theta, 2}(d, \bar{t})=-\frac{1}{36}\left(\frac{1}{d}\right)^{2}\left[\operatorname { e x p } ( - \frac { 2 } { 3 } ( l \overline { t } - d + 1 ) ) \cdot \left(B_{\theta, 2}{ }^{(1)} \cos \left(\frac{2 \sqrt{2}}{3}(l \bar{t}-d+1)\right)+\right.\right. \\
\left.\left.B_{\theta, 2}{ }^{(2)} \sin \left(\frac{2 \sqrt{2}}{3}(l \bar{t}-d+1)\right)\right)+\frac{12}{d l^{2} l_{1}}\right]
\end{gathered}
$$

for stresses in each layer, where

$$
\begin{gathered}
B_{r, 1}{ }^{(1)}=\frac{1}{6}\left(16 d^{2}-4 d-3\right) \\
B_{r, 1}{ }^{(2)}=-\frac{1}{6}\left(8 \sqrt{2} d^{2}+(8+6 \sqrt{2}) d+3 \sqrt{2}\right) \\
B_{\theta, 1}{ }^{(1)}=\frac{1}{3}\left(8 d^{2}-2 d+6\right)
\end{gathered}
$$




$$
\begin{gathered}
B_{\theta, 1}{ }^{(2)}=-\frac{1}{3}\left((18 \sqrt{2}+4) d^{2}+4 \sqrt{2} d-6 \sqrt{2}\right) \\
B_{r, 2}{ }^{(1)}=\left(\frac{2}{3}-\frac{5}{3 d}\right) \tilde{A}_{1}-\frac{2 \sqrt{2}}{3} \tilde{A}_{2} \\
B_{r, 2}{ }^{(2)}=\left(\frac{2}{3}-\frac{5}{3 d}\right) \tilde{A}_{2}+\frac{2 \sqrt{2}}{3} \tilde{A}_{1} \\
B_{\theta, 2}{ }^{(1)}=\left(\frac{2}{3}-\frac{3}{2 d}\right) \tilde{A}_{1}-\frac{2 \sqrt{2}}{3} \tilde{A}_{2} \\
B_{\theta, 2}{ }^{(2)}=\left(\frac{2}{3}-\frac{3}{2 d}\right) \tilde{A}_{2}+\frac{2 \sqrt{2}}{3} \tilde{A}_{1} .
\end{gathered}
$$

Figures 4-6 show examples of derived displacements and stresses in outer medium. This case seems to be more important due to analysis of possible damping improvement when one deals with controlled process of loading. The developed approach gives also possibility of optimization process of wave propagation outside cavity in the case of several layers with varying physical properties. This problem, however, is left for further investigation. The obtained solution combined with superposition principle leads to results concerned with short time impulse or series of such loads. Detailed analysis of this model will be presented in future works.

(a)

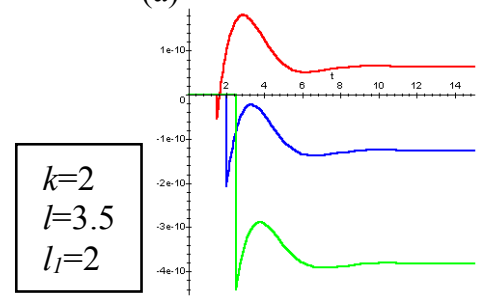

(b)

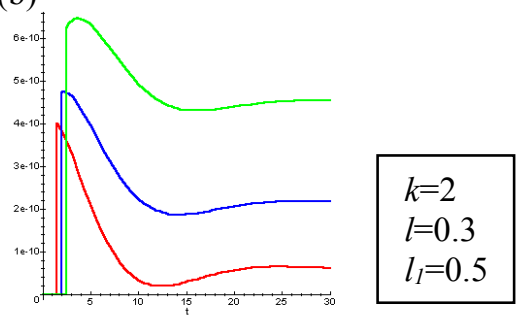

Fig. 4. Displacement $\bar{u}_{2}(t)$ in outer infinite region: $d=2.5$ (red); $d=3.0$ (blue); $d=3.5$ (green).

(a)

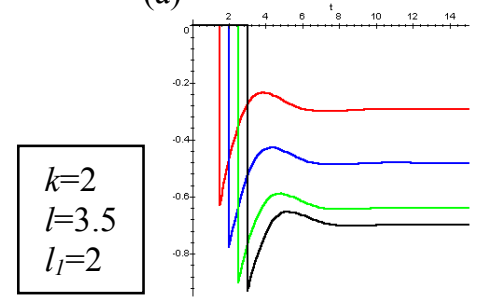

(b)

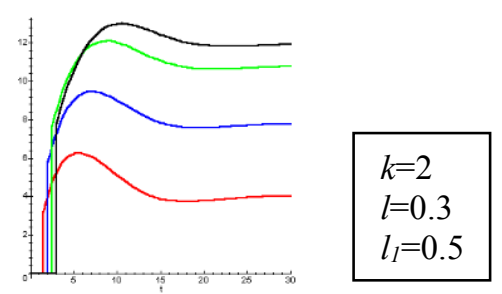

Fig. 5. Radial stress $\bar{\tau}_{r, 2}(t)$ in outer infinite region: $d=2.5$ (red); $d=3.0$ (blue); $d=3.5$ (green); $d=4.0$ (black).

One can see, e.g., that along with increase of the parameter $l_{l}$, which is related to mass densities of layers surrounding cavity (see equations (27)), damping of considered characteristics is higher, i.e. solutions stabilizes faster (it does not vanish due to considered 
pressurization represented by a load constant in time). More detailed analysis is possible and it is left for future investigations.

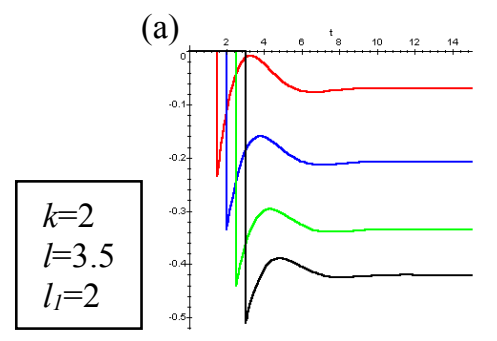

(b)

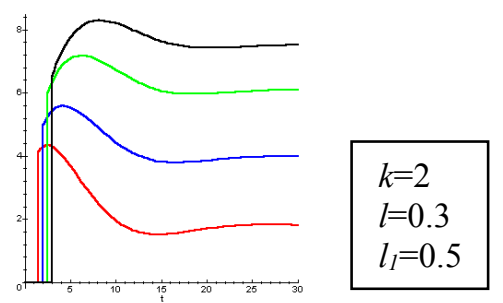

Fig. 6. Circumferential stress $\bar{\tau}_{\theta, 2}(t)$ in outer infinite region: $d=2.5$ (red); $d=3.0$ (blue); $d=3.5$ (green); $d=4.0$ (black).

\section{Conclusions}

Analytical solution for wave propagation outside spherical cavity surrounded by layer with finite thickness and immersed in unbounded medium is obtained on the basis of previously published solution for simpler model of cavity [1]. It allows detailed parametrical analysis of displacement and stresses for various loads acting on internal walls of cavity, including short time signals which can be related to sudden impact or explosion. The considered model can be easily extended to multilayer system at low computational cost. Nonlinear physical properties of layers can be introduced in further analysis. Previously developed semianalytical procedures allow solution of such extended model. This aim is left for further work.

\section{References}

1. J. D. Achenbach, North-Holland, Amsterdam, (1973)

2. P. Kozioł, Proceedings of the XIX Polish Conference "Polioptimization and CAD", Mielno, 27-28 (extended abstract), 2001

3. P. Koziol, C. Mares, SAV, 17 (4-5), 461-472 (2010)

4. P. Koziol, VDM Verlag Dr. Müller, Saarbrucken, (2010)

5. P. Koziol, J. Theor. Appl. Mech., 52, 3, 687-697 (2014)

6. G. Adomian, Kluwer Academic Publishers, Dordrecht (1989)

7. S. Mallat, Academic Press Inc. Ltd, London (1998) 